\title{
Trouble comportemental en sommeil paradoxal et maladies neurodégénératives
}

Jean-François Gagnon, Ronald B. Postuma, Jacques Yves Montplaisir

> Il existe une phase du sommeil caractérisée par une atonie musculaire, par la présence de mouvements oculaires rapides et par une activité électroencéphalographique (દદG) semblable à celle de l'éveil : c'est le sommeil paradoxal (SP). La paralysie qui caractérise le SP permet l'expression de l'activité onirique sans interrompre le déroulement du sommeil. Dès les années 1960, Michel Jouvet et ses collaborateurs ont observé chez le chat que des lésions bilatérales de régions voisines du locus coeruleus produisaient un SP sans atonie musculaire et permettaient ainsi l'observation des comportements oniriques [1]. Cependant, ce n'est qu'en 1986 que Carlos Schenck et ses collaborateurs ont décrit, chez l'humain, l'existence d'une parasomnie caractérisée par une perte partielle ou complète de l'atonie musculaire du SP et l'apparition de comportements souvent complexes et indésirables (comme parler, frapper, sauter hors du lit, rire, injurier...) au cours du SP, dont l'expression semble étroitement associée à la mise en acte du contenu de l'activité onirique qui l'accompagne [2]. Ils ont nommé cet état le trouble comportemental en sommeil paradoxal (TCSP) ou rapid-eye-movement sleep behaviour disorder (RBD).

\section{Développement}

d'une affection neurodégénérative chez les patients atteints de TCSP

Pendant longtemps, le TCSP a été considéré comme une parasomnie affectant uniquement le sommeil sans perturbation significative des activités de la vie diurne. Depuis 1996, trois groupes de recherche ont rapporté, après avoir suivi à long terme des individus atteints de TCSP, que ces derniers présentaient un risque supérieur à la moyenne de voir s'installer une affection neurodégénérative, notamment une maladie de Parkinson (MP), une démence à corps de Lewy $(D C L)$ ou une atrophie multisystémique (AMS) [3-5]. L'étude la plus récente a été effectuée dans notre laboratoire; elle a porté sur 93 patients affectés d'un TCSP idiopathique, la plus grande cohorte étudiée jusqu'à maintenant; nous avons utilisé une méthode de calcul du risque qui tient compte de l'ensemble des sujets de l'étude longitudinale [5]. Nous avons observé, après un délai d'environ cinq ans, l'apparition d'une maladie neurodégénérative chez 26 sujets: 14 souffraient de MP (dont deux avec une démence associée), sept de DCL et un de AMS. De plus, quatre patients exprimaient les critères cliniques de la maladie d'Alzheimer (MA); par conséquent, 13 patients étaient affectés d'une démence. Ceux chez lesquels un diagnostic de MA avait été posé étaient en réalité à un stade précoce de la DCL mais ils ne répondaient pas, à ce stade, à tous les critères diagnostiques de la $D C L$, comme la présence d'un parkinsonisme, d'hallucinations visuelles ou d'une fluctuation de la cognition et de la vigilance. Ils avaient donc été considérés provisoirement comme atteints de MA mais le suivi a confirmé qu'il s'agissait probablement d'une DCL. II est intéressant de noter que le TCSP est rare dans la MA [6]. Ainsi, l'identification d'un TCSP chez
J.F. Gagnon, J.Y. Montplaisir :

Centre d'étude du sommeil et des rythmes biologiques, Hôpital du Sacré-Cœur de Montréal, 5400 , boulevard Gouin ouest, Montréal, Québec, H4J 1C5 Canada.

jy.montplaisir@umontreal.ca

R.B. Postuma : Département de neurologie, L7-305, Hôpital Général Montréal,

1650, avenue Cedar, Montréal, Québec, H3G IA4 Canada.

un individu suspecté d'être atteint d'une démence pourrait faciliter le diagnostic différentiel entre la MA et la DCL.

\section{Marqueurs précoces}

de maladies neurodégénératives chez les patients atteints de TCSP

Les maladies neurodégénératives débutent rarement de façon subite. II existe une phase silencieuse au cours de laquelle le seuil de diagnostic clinique n'est pas franchi. Dans la MP et la $D C L$, l'atteinte des systèmes neuronaux débuterait bien avant l'apparition des symptômes cliniques dans les régions du tronc cérébral qui régissent l'atonie musculaire du SP. Ceci expliquerait pourquoi le TCSP est souvent l'un des symptômes initiaux de la MP et de la DCL. C'est pourquoi nous avons recherché des marqueurs précoces de maladies neurodégénératives, en particulier ceux de la MP et de la DCL, chez nos patients affectés d'un TCSP idiopathique. Nous avons alors observé un ralentissement moteur mesuré par des épreuves chronométrées, des troubles visuels et olfactifs, des troubles autonomiques, de même qu'un ralentissement de l'activité દદG à l'éveil avec une atteinte des fonctions cognitives évaluées par des épreuves neuropsychologiques $[7,8]$. L'ensemble de ces résultats montre que, chez un grand nombre de malades, le TCSP représente le stade précoce d'une maladie neurodégénérative; elle se profile donc comme une cible privilégiée pour 
les traitements de neuroprotection qui seront éventuellement disponibles.

\section{TCSP et Maladie de Parkinson}

Parallèlement, nous avons étudié le TCSP dans des populations de sujets ayant été diagnostiqués comme atteints de MP. Nous avons d'abord dûment noté que plus du tiers des sujets parkinsoniens étaient affligés d'un TCSP [9]. Nous avons ensuite observé que seuls les sujets parkinsoniens victimes d'un TCSP présentaient un ralentissement de l'activité દદG à l'éveil et une atteinte des fonctions cognitives détectée lors des tests neuropsychologiques $[10,11]$. Les sujets parkinsoniens non affectés de TCSP ne présentaient ni anomalies de l'દદG, ni atteintes des fonctions cognitives. Ces phénotypes distincts suggèrent qu'il existe une distribution topographique ou une sévérité des pertes neuronales différentes entre les patients parkinsoniens selon qu'ils sont affligés ou non d'un TCSP.

\section{Conclusion}

Dans l'ensemble, ces travaux laissent envisager que le TCSP est bien plus qu'une maladie du sommeil. Un nombre important d'individus qui souffrent de TCSP montrent, entre autres, une détérioration de leurs capacités cognitives qui évolue souvent vers une démence. Le suivi à long terme des patients atteints de TCSP nous permettra peut-être de mieux comprendre les facteurs qui risquent de provoquer certaines maladies neurodégénératives. $\diamond$

Neurodegenerative

disorder in idiopathic REM

sleep behaviour disorder

\section{CONFLIT D'INTÉRÊTS}

Les auteurs déclarent n'avoir aucun conflit d'intérêts concernant les données publiées dans cet article.

\section{RÉFÉRENCES}

1. Jouvet M, Delorme F. Locus coeruleus et sommeil paradoxal. CR Soc Biol 1965; 159 : 895-9.

2. Schenck CH, Bundlie SR, Ettinger MG, Mahowald MW Chronic behavioral disorders of human REM sleep: a new category of parasomnia. Sleep $1986 ; 9: 293-308$
3. Schenck CH, Bundlie SR, Mahowald MW. Delayed emergence of a parkinsonian disorder in $38 \%$ of 29 older men initially diagnosed with idiopathic rapid eye movement sleep behaviour disorder. Neurology $1996 ; 46: 388-93$

4. Iranzo A, Molinuevo JL, Santamaría J, et al. Rapideye-movement sleep behaviour disorder as an early marker for a neurodegenerative disorder: a descriptive study. Lancet Neurol 2006 ; 5 : 572-7.

5. Postuma RB, Gagnon JF, Vendette M, et al. Quantifying the risk of neurodegenerative disease in idiopathic REM sleep behavior disorder. Neurology 2009 ; 72: 1294-5.

6. Gagnon JF, Petit D, Fantini ML, et al. REM sleep behavior disorder and REM sleep without atonia in probable Alzheimer disease. Sleep 2006 ; 29 : 1321-5.

7. Postuma RB, Lang AE, Massicotte-Marquez J, Montplaisir J. Potential early markers of Parkinson disease in idiopathic REM sleep behavior disorder. Neurology 2006 ; 66 : 845-51.

8. Massicotte-Marquez J, Décary A, Gagnon JF, et al. Executive dysfunction and memory impairment in idiopathic REM sleep behavior disorder. Neurology 2008; $70: 1250-7$.

9. Gagnon JF, Bédard MA, Fantini ML, et al. REM sleep behavior disorder and REM sleep without atonia in Parkinson's disease. Neurology 2002 ; 59 : 585-9.

10. Gagnon JF, Fantini ML, Bédard MA, et al. Association between waking દદG slowing and REM sleep behavior disorder in PD without dementia. Neurology 2004 ; 62: 401-6.

11. Vendette M, Gagnon JF, Décary A, et al. REM sleep behavior disorder predicts cognitive impairment in Parkinson disease without dementia. Neurology 2007 69 : 1843-9.

\section{NOUVELL}

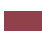 \\ Les protéines ARF \\ Interrupteurs moléculaires \\ de la prolifération des tumeurs et de la formation des métastases}

Pierre-Luc Boulay, Audrey Claing
Université de Montréal,

Département de pharmacologie, CP 6128, Succursale Centre-ville, Montréal (Québec), H3C 3J7 Canada. audrey.claing@umontreal.ca
$>$ Le cancer du sein est la forme de cancer la plus fréquente chez la femme. Le haut niveau d'expression et/ou d'activation de plusieurs récepteurs à activité tyrosine kinase comme le récepteur du facteur de croissance épidermique (epidermal growth factor receptor: EGFR) est une caractéristique des cancers invasifs, dit agressifs et également l'indication d'un mauvais pronostic. Afin de former des métastases, les cellules cancéreuses doivent pouvoir migrer (Figure 1). Ce processus implique l'activation de plusieurs voies de signalisation qui vont induire un remodelage du cytosquelette d'actine. Plusieurs protéines intracellulaires régissent la prolifération, la migration et la capacité invasive des cellules cancéreuses. L'oncogène RAS est l'un des gènes les plus importants dans l'apparition des cancers puisqu'environ 20 à $30 \%$ des tumeurs possèdent une mutation pour cette protéine $\mathrm{G}$ monomérique [3]. La superfamille des Ras se divise en 6 groupes: Ras, Rho, Rab, Ran, Rad et ARF
[4]. En particulier, le rôle des protéines ARF (ADP-ribosylation factors) dans cette réponse cellulaire est maintenant reconnu $[1,2]$. Nous avons donc émis l'hypothèse que les ARF sont des facteurs importants facilitant la migration cellulaire stimulée par l'EGF et par conséquent, des protéines clés dans la progression du cancer.

\section{Les GTPases de la famille des ARF}

Six gènes encodent les protéines ARF (l à 6). Les isoformes ARFl à ARF5 